\title{
La Suada Gallicana (1679) et son « double spécimen d'analyse logico-rhétorique »
}

\section{Giuliano Ferretti et Francis Goyet}

\section{(2) OpenEdition}

1 Journals

Édition électronique

URL : http://journals.openedition.org/rhetorique/330

DOI : 10.4000/rhetorique.330

ISSN : 2270-6909

Éditeur

UGA Éditions/Université Grenoble Alpes

Édition imprimée

ISBN : 978-2-84310-274-5

Référence électronique

Giuliano Ferretti et Francis Goyet, « La Suada Gallicana (1679) et son « double spécimen d'analyse logico-rhétorique » », Exercices de rhétorique [En ligne], 3 | 2014, mis en ligne le 12 juin 2014, consulté le 12 septembre 2020. URL : http://journals.openedition.org/rhetorique/330 ; DOI : https://doi.org/ 10.4000/rhetorique.330

Ce document a été généré automatiquement le 12 septembre 2020.

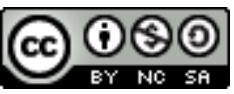

Les contenus de la revue Exercices de rhétorique sont mis à disposition selon les termes de la Licence Creative Commons Attribution - Pas d'Utilisation Commerciale - Partage dans les Mêmes Conditions 4.0 International. 


\title{
La Suada Gallicana (1679) et son « double spécimen d'analyse logico- rhétorique »
}

\author{
Giuliano Ferretti et Francis Goyet
}

1 Nous publions ici deux analyses rhétoriques détaillées, par un professeur de rhétorique peu connu, Abraham Vechner (1636-1704). Ces analyses ont constitué, avec quelques autres par Melchior Junius (1545-1604), le corpus d'un séminaire de Master que nous avons tenu ensemble pendant trois années consécutives, de 2006 à 2009, sur le thème «rhétorique et histoire ». Junius en 1598 analyse des discours qu'il trouve chez des historiens du $\mathrm{XVI}^{\mathrm{e}}$ siècle, par exemple Paolo Giovio ou les frères Du Bellay, ce qui est exceptionnel à son époque ${ }^{1}$. Vechner en 1679 a le même geste, tout aussi exceptionnel ${ }^{2}$ : il tire deux discours de l'Historia de Jacques-Auguste de Thou (1553-1617), discours qui ont été effectivement tenus. Le premier a en effet été prononcé en 1567 par François de Coligny d'Andelot, et le second l'a été en 1600 par Guillaume Du Vair. Nous sommes ainsi au plus près de l'événement historique. Le contraste est grand avec les commentaires rhétoriques des $\mathrm{XVI}^{\mathrm{e}}$ et $\mathrm{XVII}^{\mathrm{e}}$ siècles portant sur des discours de l'Antiquité. Dans ce cas-là, non seulement le commentateur n'est pas un contemporain, mais les discours sont eux-mêmes des reconstitutions dues à l'historien antique qui nous les a transmis.

2 Nous donnerons un aperçu d'abord de la carrière de Vechner, puis de son seul livre, la Suada Gallicana (L'Éloquence française) de 1679, enfin du type d'analyse qu'il pratique, « logico-rhétorique ».

\section{La carrière d'Abraham Vechner (1636-1704)}

3 La carrière de Vechner se déroule à peu près entièrement au Gymnasium de Görlitz, sur la rive gauche de la Neisse, donc dans l'Allemagne actuelle ${ }^{3}$. Cette ville de l'Est de la Saxe est alors luthérienne, comme toute sa région, la Lusace ${ }^{4}$. Nous nous appuierons sur deux ouvrages: Christian Funcke (1626-1695), Doctrinae nec non Disciplinae Augusti 
Gymnasii Gorlic., programme des études très détaillé5 ; Christian Knauth (1706-1784), Das Gymnasium Augustum zu Görlitz, Görlitz, Fickelscherer, 1765, p. 98-996.

Le père de notre auteur, le théologien David Vechner (1594-1669), est de 1633 à 1665 recteur à vie du Gymnasium 7 . Abraham Vechner lui-même est en 1667 doyen à Sprottau, ou Szprotawa, ville proche, mais de l'autre côté de la Neisse ${ }^{8}$. Ensuite, il est au Gymnasium, sous le rectorat suivant (1665-1695), celui de Funcke déjà nommé, lequel fait d'emblée, en matière de rhétorique, une large place à Vossius ${ }^{9}$. Vechner monte la hiérarchie qui va de Conrector (co-recteur, en 1669) à Prorector (pro-recteur, en 1671 ${ }^{10}$ ) ; enfin, il résigne cette dernière charge en 1687 , pour cause de cécité. Comme prorecteur, il enseigne en seconde la poétique et des éléments de rhétorique, et en première la rhétorique ${ }^{11}$. La classe de première était alors la classe la plus haute, et l'élève y restait deux ou même, à Görlitz, trois années (Doctrinae, p. 39), ce qui fait de cette " première » une propédeutique relevant déjà de l'enseignement supérieur, dont l'ambiance studieuse évoque, plus que le lycée, les actuelles classes préparatoires françaises.

\section{La Suada Gallicana (1679), ou L'Éloquence française}

5 La Suada est le seul ouvrage de Vechner ${ }^{12}$. C'est un gros recueil de discours historiques, c'est-à-dire ce que l'époque nomme depuis Henri Estienne en 1570 un conciones. C'est aussi le seul conciones que nous connaissions qui n'ait que des discours modernes: quatre-vingt onze au total, dont certains redonnés ensuite en traduction allemande ${ }^{13}$. Enfin, ces discours sont tous tirés du même auteur, de Thou, ce qui constitue un autre trait original, puisqu'un conciones est d'habitude une anthologie de discours tirés d'historiens divers, antiques en général (et modernes, chez Junius). Dans son avis au lecteur ${ }^{14}$, Vechner annonce qu'il souhaiterait constituer d'autres volumes semblables, avec des discours tirés des historiens Gramond, Guichardin, Giovio, Strada, Sleidan et bien d'autres. Il ne dit pas pourquoi il a commencé par de Thou, mais c'est évident. L' Histoire universelle de celui-ci a suscité tout au long $\mathrm{du}_{\mathrm{XVII}}{ }^{\mathrm{e}}$ siècle un très vif intérêt, en Allemagne en particulier, avec l'espèce de feuilleton qu'a été sa publication étalée dans le temps, jusqu'en 1733 (ce que Vechner nomme la « Continuation »).

6 Le titre de l'unique édition est, selon l'usage de l'époque, tout un programme : Suada Gallicana, h. e. conciones et orationes Thuanaeae, Una cum Argumentis praefixis [...] ex [...] Historiarum continuatione excerptarum Orationum; Uti et Teutonicorum quarundam appendice: Prudentiae Civilis quoddam veluti tameion exhibentes. Accedit Analyseos LogicoRhetorico Specimen duplex [...], Francfort et Leipzig ${ }^{15}$, J. Adam Kästner, 1679. Soit: L'Éloquence française, c'est-à-dire harangues et discours de de Thou, précédés d'un argument, et tirés de la continuation de son Histoire universelle; ainsi que quelques-uns traduits en allemand: lesquels montrent la prudence politique à la manière dont on montre dans une exposition les pièces d'un trésor. S'y ajoute un double spécimen d'analyse logico-rhétorique. Notons d'abord que suadus est un adjectif : il faut donc sous-entendre ars, ou peut-être methodus. À sa p. 1, Vechner récrit son titre en Peitho Gallicana (Peithô est, en grec, la déesse de la persuasion). On pourrait donc retraduire : L'art français de persuader - un art « français » mais écrit en latin, langue qui est aussi celle de de Thou ${ }^{16}$. D'autre part, le seul mot du titre qui soit en caractères grecs, tameion (et non tiameion, plus usuel), signe que c'est un renvoi à un passage topique de Plutarque : «la mémoire des enfants 
[...] est comme le trésor de l'éducation [tès paideias tameion] » (De l'éducation des enfants, $\S 13,9 \mathrm{D}-\mathrm{F})$.

7 La fin du passage de Plutarque fait le lien avec le monde moderne : "Que les pères ne perdent pas non plus de vue que la part de la mémoire dans les études ne sert pas seulement pour l'éducation, mais encore dans une proportion non négligeable pour les activités de la vie [tas tou biou praxeis]. Car la mémoire des actions passées devient un modèle pour décider sagement des actions à venir. » Décider sagement, c'est le thème de la «prudentia civilis » mis au titre de la Suada. La prudentia ou sagesse et intelligence politiques est aussi la qualité attribuée à tous les dédicataires de l'ouvrage, énumérés dans le début de la préface : le Duc de Saxe et les Grands, puis tous ceux qui gouvernent Görlitz, «consuls, préteurs, syndic, secrétaire, jurats, sénateurs ${ }^{17}$ ». Tous sont loués pour leur prudentia éminente dans le gouvernement des affaires. C'est redit à la fin de la préface ${ }^{18}$ avec un mot mis en caractères grecs : ils sont "politikôtatoi ", ils ont le sens politique, le sens de l'État, de la Cité.

8 Misant sur la modernité, publiée à Francfort et Leipzig, la Suada visait sans doute le public général, et pas seulement scolaire. Nous ne savons pas quelle a été sa réception, mais elle semble avoir été modeste : une simple mention signalétique dans Le Journal des sçavans (1680, vol. 7, p. 577), et une mise à l'Index en 1683, pour la seule raison, presque automatique, que les discours qu'y reprend Vechner « sont, avec quelques extraits dans un volume par Lampadius, les seules portions de l'œuvre de de Thou à être encore condamnées " par Rome ${ }^{19}$. La Suada n'est même pas citée parmi les ouvrages au programme de son propre Gymnasium, le Doctrinae de Funcke (que Knauth, p. 102, date de 1686) prescrivant un autre conciones, qui n'a classiquement que des discours d'historiens antiques ${ }^{20}$. Ce silence ne prouve pas en soi que la Suada n'ait pas été utilisée en classe. Le programme publié étant aussi un outil de communication, il affiche surtout des auteurs latins et grecs. On peut également supposer que la Suada relevait des cours "privés » de Vechner, ce tutorat payant en petit cercle, par définition hors programme. Même si le but y restait de préparer les élèves ou plutôt étudiants aux cours officiels, ce cadre plus souple permettait des expérimentations pédagogiques, proche de ce que nous appellerions aujourd'hui de la recherche universitaire. La Suada et ses deux analyses logico-rhétoriques ont peut-être un ton plus ludique qu'il n'y paraît à première vue : l'enseignant s'y fait profondément plaisir, ce qui inclut le plaisir de susciter celui de ses étudiants.

\section{L'analyse « logico-rhétorique » de deux discours}

9 La Suada ne contient donc que deux analyses détaillées, même si on trouve, en tête de la plupart des discours de de Thou, des résumés ou arguments qui sont bien souvent un premier repérage rhétorique. L'étroitesse du corpus n'a pas facilité notre travail de décryptage. Un plus grand nombre d'analyses détaillées nous aurait permis de dégager des régularités avec quelque certitude. À défaut, notre traduction est à prendre comme une proposition, le résultat d'hypothèses, argumentées dans nos notes. Heureusement pour nous, Vechner a le souci constant de doubler presque chaque terme qu'il emploie par un autre, plus habituel dans la terminologie rhétorique.

En fait de décryptage, nous soulignerons ici seulement deux grandes généralités : le distinguo entre « logique » et " rhétorique », et le fait de conjoindre deux discours, qui font la paire. 
11 La formule logico-rhétorique renvoie certainement au distinguo posé par Ramus (1515-1572) et usuel ensuite en Allemagne : d'un côté la rhétorique, c'est-à-dire le style, l'elocutio; de l'autre la logique, c'est-à-dire l'argumentation (la dialectique, chez Ramus). Appartiennent donc à la rhétorique en ce sens restreint les "ornements ", le relevé des figures de style par lequel Vechner ponctue chacun de ses développements. Tout ce qui précède ce relevé appartient en revanche à la « logique ». Cet intérêt pour l'argumentation est souligné de façon spectaculaire par le fait que l'analyse ne suit pas l'ordre du texte. Vechner analyse ou découpe chacun de ses deux discours en trois parties : I. exorde, II. propositio, III. argumentation ; s'y ajoute, ou non, une conclusion. Or, seul l'exorde se trouve à la même place (le début) dans le texte comme dans l'analyse. Pour le reste, le texte du premier discours se présente sous l'ordre suivant: III. argumentation, II. propositio, ce II. étant lui-même suivi de la suite et fin du $4^{\mathrm{e}}$ et dernier argument, donc de la fin de III. De même, le second discours a, dans l'ordre : III. argumentation, II. propositio, ce II. étant là encore suivi d'un $3^{\mathrm{e}}$ et dernier argument. La « logique » consiste donc d'abord, pour Vechner, à repérer où est la propositio ou thèse à défendre, et il la trouve à l'intérieur même de l'argumentation. À partir de là, il identifie les arguments à l'appui de la thèse. Il cherche donc la structure profonde du texte, quitte à malmener le découpage de surface.

12 L'autre élément qui appelle la réflexion est l'annonce d'un "double » spécimen d'analyses, "duplex ». Vechner ne se contente pas de donner l'analyse de deux discours. Il a choisi ces deux discours dans des genres distincts, comme il le dit dans le peu qu'il donne en guise de justification de son spécimen ${ }^{21}:$ "C'est en pensant plus spécialement aux Élèves que nous avons trouvé bon d'ajouter un spécimen d'analyses logicorhétoriques sur deux discours, chacun dans un genre de causes différent [ou " opposé ", «diverso »]. » De fait, le premier des deux discours relève du genre délibératif et le second, de l'épidictique. Il semble évident que Vechner a construit un parallèle terme à terme, une comparatio entre ses deux discours. Le premier appelle à la guerre : le chef protestant Coligny d'Andelot lance la deuxième guerre de religion, en 1567. Le second discours, lui, parle de paix : en épousant Henri IV, Marie de Médicis va donner un héritier à la Couronne et par là éloigner le spectre d'un retour des mêmes guerres de religion.

En séminaire, nous nous sommes demandé si de là on pouvait pousser plus loin l'interprétation. Il se pourrait par exemple que l'opposition entre les deux discours se traduise, dans l'analyse, par une opposition entre causa et gratia, puisque le discours épidictique est dit être "syncharistique ", sur le grec kharis, la grâce. Dans le discours d'appel à la guerre domine la causa, ce mot signifiant en particulier la responsabilité personnelle. Toute la question y est en effet de savoir à qui remonte la responsabilité de la situation présente, et donc de la guerre qui s'ensuivra. Il s'agit de nommer le premier fauteur de la violence, celui que l'on pourra "accuser», verbe formé sur causa. La traduction Du Ryer le fait sentir, dans le $4^{\mathrm{e}}$ argument (nous soulignons) : "Nous ne devons pas nous mettre en peine de ce que diront nos Ennemis, et les méchants, comme si nous avions été cause d'une guerre pernicieuse. En effet, il n'en faut accuser qu'euxmêmes ». Par avance d'Andelot rejette la responsabilité sur le camp adverse - rejet qui est classiquement la translatio criminis. La guerre est ainsi un monde du « à cause de qui », du "à qui la faute ». Le discours de paix, lui, serait par comparaison dans le monde de la grâce, culminant avec l'évocation de la grâce divine en amont de tout. " Grâce à Dieu », et non « à cause de Dieu », la reine est bien arrivée à Marseille. Grâce à 
Dieu, elle aura des enfants. Grâce à Dieu, et à elle-même, parce qu'elle voudra bien répondre à sa "vocation", le royaume évitera une nouvelle guerre civile, etc. C'est le même mouvement de remontée que celui qui amène à identifier le fauteur de guerre ou causa, mais on est dans une autre planète, dans le monde de la grâce, monde d'ailleurs hanté par le spectre du retour à la guerre civile.

On voit que, pour pouvoir confirmer une telle hypothèse, il faudrait en savoir plus sur l'emploi du mot causa dans la culture rhétorique et philosophique, voire théologique, du temps de Vechner, en Allemagne ou plutôt en Saxe. Cela dépasse nos compétences actuelles. Pour autant, et pour conclure ce bref aperçu d'ensemble, le double spécimen ne manque pas d'être très suggestif, ne serait-ce que par la beauté des discours analysés. Junius note, dans la préface de son propre conciones, que l'introduction de discours modernes a beaucoup plu à ses étudiants. Nous avons fait la même expérience avec nos propres étudiants. C'est d'ailleurs pourquoi nous donnons en annexe tout le récit par lequel de Thou encadre le premier des deux discours. Car la seule lecture cursive d'un historien de ce type est déjà une expérience rhétorique, qui donne à voir très concrètement ce que peut signifier, dans une situation historique et non fictive, la puissance de la parole, et à quel point un discours peut être à part entière un événement, une action comme une autre, et non un ornement inséré.

\section{NOTES}

1. Dans son Orationum ex historicis tam veteribus, quam recentioribus..., Strasbourg, Zetzner, sur le modèle de son Epistolae ex historicis, tam veteribus, quam recentioribus..., Montbéliard, Zetzner, 1595 (lettres tirées d'historiens antiques et modernes). Voir l'analyse d'un discours que tire Junius de Buchanan (Rerum Scoticarum Historia, 1582) dans : Francis Goyet, «Le discours de (re)conciliatio (Junius, 1598) : enjeux politiques d'une analyse rhétorique ", L'art de la conciliation, éd. S. Cornic et P. Servet, Genève, Droz (Cahiers du Gadges, $\left.n^{\circ} 11\right)$, 2013, p. 91-111.

2. Un cas proche est Christoph Schrader (1601-1680), Hypotheses oratoriae, Helmstedt, Müller, 1669, http://reader.digitale-sammlungen.de/resolve/display/bsb10154236.html. Celui-ci y donne, avec leurs corrigés, des sujets de discours, qu'il tire du De statu religionis, 1556, de Jean Sleidan (1506-1556). Cette histoire des troubles de religion en Allemagne sous Charles Quint suscite, dit Schrader, un vif intérêt chez ses étudiants. Sur le contexte pédagogique de son utilisation, voir Jean-Luc Le Cam, La politique scolaire d'Auguste le Jeune de Brunswick-Wolfenbüttel et l'inspecteur Christoph Schrader 1635-1666/80, Wiesbaden, Harrassowitz, 1996, vol. I, p. 162-186, en particulier p. 172-175.

3. Nous remercions vivement Jean-Luc Le Cam d'avoir relu et complété notre texte, et de confirmer notre sentiment que l'enseignement dans ce lycée était de très haute qualité, même pour l'Allemagne.

4. La situation religieuse a été plus compliquée auparavant : Pierre Béhar, «La Lusace du début du XVII ${ }^{\mathrm{e}}$ siècle et la doctrine de Jacob Bœhme ", Les frontières religieuses en Europe $d u X^{e}$ au XVII siècle, éd. A. Ducellier et al., Paris, Vrin, 1992, p. 159-169.

5. Sans lieu ni date, non paginé; nous renvoyons aux pages du pdf, URL : http://digital.slubdresden.de/werkansicht/dlf/24819/1/cache.off 
6. URL : http://www.mdz-nbn-resolving.de/urn/resolver.pl?urn=urn:nbn:de:bvb:12bsb10000953-5. On peut ajouter: Titus Wilde, Neues lausitzisches Magazin, Görlitz, Müller, 1864, volumes 41-42, p. 113 (sur Google Livres).

7. Knauth, p. 93-97. Il a été en relation amicale avec Comenius et s'est inspiré de ses méthodes. Son père est «le célèbre D. George Vechner» (Knauth, p. 93), Professor Pietatis à Beuthen, puis pasteur et superintendant à Brieg. Nous ne savons pas s'il y a un lien avec le médecin Abraham Vechner (qui a de nombreuses publications à Marburg entre 1609 et 1620) ni avec Daniel Vechner (1572-vers 1632), professeur de rhétorique à Goldberg. Ce dernier est le seul Vechner à être mentionné par L. D. Green et J. J. Murphy, Renaissance Rhetoric Short-Title Catalogue 1460-1700 (Aldershot, Ashgate, 2006), qui citent de lui Amplificationum rhetoricarum..., Breslau (Wroclaw), 1614, et Figurarum rhetoricarum methodus, Leipzig, 1621.

8. Selon les titres de deux «Festschrift » en son honneur: Applausus Votivus..., Wittenberg, Hake, 1667, p. 4 ; Patronorum venerandorum..., Wittenberg, Henckel, 1667, p. 4.

9. Funcke, Gymnasii Gorl. Doctrina, Görlitz, Zipper, 1667 (p. 7 ; cité par Knauth, p. 100). Vechner (Suada, préface, p. A5 $\mathrm{r}^{\circ}$ ) renvoie, lui, au De Arte Historica de Vossius, chap. $\mathrm{xx}$, en faveur de l'insertion de discours dans le genre historique.

10. Titre qu'il se donne lui-même dans la préface de la Suada. Selon Knauth, il ne le serait qu'en 1681 : lapsus probable, corrigé par Wilde, pour 1671.

11. Selon le Doctrinae (p.12), en première, le recteur (Funcke) enseigne logique, philologie, théologie et des éléments de philosophie ; le pro-recteur (Vechner), « Latinam Linguam, Rhetoricam \& Oratoriam "; le co-recteur, grec, histoire et arithmétique; le sub-rector, hébreu et poésie. En seconde (ibid.), le pro-recteur enseigne les «Elementa Poëtica \& Rhetorica» ainsi que l'historien Cornelius Nepos; d'où un traité de versification par Vechner, que cite le Doctrinae (« Stichopoeiam s. Versificatoriam VECHNERI ", p. 27 et 30) et que nous n'avons pas retrouvé.

12. Numérisé par la StaBi (voir le lien en tête de chaque discours). Autres publications : le traité de versification introuvable (note précédente); l'édition, en 1673, d'un ouvrage de son père, Universae Germaniae breviarium (déjà édité par son père : Görlitz, Hermann, 1645, Google) ; Actum Progymnasticum..., Görlitz, Zipper, p. 6, 1672, où il est dit « pro-rector »; une pièce liminaire dans Funcke, Theosophico-Physiologica Cometoscopia, Görlitz, Zipper, 1682.

13. Funcke est membre de la Fruchtbringende Gesellschaft, société qui travaille à promouvoir l'allemand comme langue littéraire et poétique. Le Doctrinae (p. 4-5) mentionne la traduction du latin à l'allemand et vice-versa comme l'un des « Exercitia styli » dont le pro-recteur a la charge en première.

14. À la p. [A10] vo .

15. La page en vis-à-vis de la page de titre donne comme lieu d'édition «Görlitz », chez le même Kästner.

16. Trois ouvrages antérieurs sont intitulés Suada: Suada attica, 1615, recueil de discours de Puteanus (Google); Suada delphica, 1650, des discours de Jacob Crucius ou Jacques de Crucque (Google) ; Suada gothana c'est-à-dire allemande, 1657, des discours d'Andreas Wilke.

17. À la p. [A3] vo .

18. À la p. [A7] $\mathrm{v}^{\circ}$.

19. Samuel Kinser, The works of Jacques-Auguste de Thou, La Haye, Nijhoff, 1966, p. 304.

20. Joachim Gesenius (recteur à Leipzig, mort en 1675), Conciones civiles..., Leipzig, Fuhrmann et Breuer, 1674 (le Doctrinae, p. 6, cite l'éd. Francfort, 1683).

21. Avis au lecteur, p. [A10] $\mathrm{v}^{\circ}$-[A11] $\mathrm{r}^{\circ}$ : «In gratiam autem Scholasticae potissimum Juventutis Specimen aliquod Logico-Rhetoricae duarum e diverso Caussarum genere Orationum Analyseos addere haud displicuit. » 


\section{AUTEURS}

\section{GIULIANO FERRETTI}

Université Grenoble Alpes - RARE Rhétorique de l'Antiquité à la Révolution

\section{FRANCIS GOYET}

Univ. Grenoble-Alpes - RARE Rhétorique de l'Antiquité à la Révolution 